\title{
High prevalence of bla $\beta$-lactamase genes in Enterobacteriaceae isolates from Emilia-Romagna, Italy
}

The production of extended-spectrum $\beta$-lactamases (ESBLs) by members of the Enterobacteriaceae is an increasing challenge in the treatment of infections. The ESBL type that has been detected most frequently over the last few years is CTX$\mathrm{M}$, which also has the particular ability to spread in the community (Pitout \& Laupland, 2008). In Emilia-Romagna, a northern Italian region with a population of about 4 million, the rate of resistance to $\beta$-lactamase due to ESBLs increased rapidly in the period 2003-2006 (Gagliotti et al., 2008a). No data on the type of circulating ESBL are available at the regional level. This study aims to describe the epidemiology of ESBLs by testing a sample of clinical ESBL-producing strains that were isolated in Emilia-Romagna.

Thirty isolates were randomly selected from the strain collection of a previous study, conducted in Emilia-Romagna in 2006, to evaluate the accuracy of automated systems in detecting ESBLs in Escherichia coli, Klebsiella pneumoniae and Proteus mirabilis. These isolates were collected in four of the five laboratories participating in the study (Gagliotti et al., 2008b). The ESBL phenotype was confirmed by the combination disc method (CLSI, 2007). All strains underwent PCR amplification of $b l a_{\mathrm{TEM}}$, $b l a_{\mathrm{SHV}}, b l a_{\mathrm{CTX}-\mathrm{M}}$ and ampC-type genes. Sequencing was performed to identify the subclasses of $b l a_{\mathrm{TEM}}, b l a_{\mathrm{SHV}}$ and $b l a_{\mathrm{CTX}-\mathrm{M}}$ genes (Al Naiemi et al., 2006; D'Andrea et al., 2006; Perilli et al., 2002).

The 30 isolates studied were all clinical samples (26 E. coli, 2 K. pneumoniae and 2 P. mirabilis). Sequencing of ESBLencoding genes showed that most strains $(26 / 30 ; 87 \%)$ carried $b l a_{\text {CTX-M-1-group }}$ genes, with $b l a_{\mathrm{CTX}-\mathrm{M}-15}$ and $b l a_{\mathrm{CTX}-\mathrm{M}-1}$ being the most prevalent variants $(63 \%$ and $20 \%$, respectively) whereas the $b l a_{\text {CTХ-М-32 }}$ gene was carried by only one strain $(3 \%)$ of E. coli. The GenBank/EMBL/DDBJ accession numbers for the $b l a_{\mathrm{CTX}-\mathrm{M}-15}$, $b l a_{\mathrm{CTX}-\mathrm{M}-1}$ and $b l a_{\mathrm{CTX}-\mathrm{M}-32}$ gene sequences of E. coli strains are AY0444436, X92506 and AJ557142, respectively.

Two E. coli isolates harboured $b l_{\mathrm{SHV}-5}$ genes while one K. pneumoniae isolate had multiple ESBL genes $\left(b l a_{\mathrm{CTX}-\mathrm{M}-15}+b l a_{\mathrm{SHV}-12}\right)$. Both $P$. mirabilis isolates harboured $b l a_{\mathrm{TEM}-1}$ (non-ESBL $\beta$-lactamase gene), but the ESBL encoding genes were not identified. $b l a_{\mathrm{TEM}-1}$ was also retrieved in three E. coli isolates, while only one isolate showed an ampC genotype (CMY-2-like). Most patients had a urine infection $(87 \%)$, were $\geqslant 80$ years $(53 \%)$ and female $(60 \%)$. A high proportion $(43 \%)$ of clinical samples were collected from outpatients (Table 1). The total prevalence of resistance to ciprofloxacin and gentamicin was $80 \%$ and $30 \%$, respectively; $23 \%$ of isolates (five $E$. coli, one $K$. pneumoniae and one $P$. mirabilis) were co-resistant to both agents. The data show that CTX-M is, by far, the most common ESBL type in members of

Table 1. Genotype, sample and patient characteristics for 30 ESBL-producing strains

\begin{tabular}{|c|c|c|c|c|}
\hline & $\begin{array}{c}\text { E. coli } \\
(n=26)\end{array}$ & $\begin{array}{c}\text { K. pneumoniae } \\
(n=2)\end{array}$ & $\begin{array}{l}\text { P. mirabilis } \\
(n=2)\end{array}$ & $\begin{array}{c}\text { All organisms } \\
\quad(n=30)\end{array}$ \\
\hline \multicolumn{5}{|l|}{ ESBL type } \\
\hline CTX-M-15 & $17^{\star}$ & 1 & 0 & $18(60 \%)$ \\
\hline CTX-M-1 & $6^{*}$ & 0 & 0 & $6(20 \%)$ \\
\hline CTX-M-32 & 1 & 0 & 0 & $1(3 \%)$ \\
\hline CTX-M-15 + SHV-12 & 0 & 1 & 0 & $1(3 \%)$ \\
\hline SHV-5 & $2 \dagger$ & 0 & 0 & $2(7 \%)$ \\
\hline Not identified & 0 & 0 & $2 \ddagger$ & $2(7 \%)$ \\
\hline \multicolumn{5}{|l|}{ Sample } \\
\hline Urine & 22 & 2 & 2 & $26(87 \%)$ \\
\hline Other/unknown & 4 & 0 & 0 & $4(13 \%)$ \\
\hline \multicolumn{5}{|l|}{ Patient } \\
\hline Outpatient & 12 & 0 & 1 & $13(43 \%)$ \\
\hline Inpatient (hospital) & 11 & 1 & 0 & $12(40 \%)$ \\
\hline $\begin{array}{l}\text { Inpatient (long-term } \\
\text { facilities) }\end{array}$ & 2 & 1 & 1 & $4(13 \%)$ \\
\hline Unknown & 1 & 0 & 0 & $1(3 \%)$ \\
\hline \multicolumn{5}{|l|}{ Age category (years) } \\
\hline $0-19$ & 1 & 0 & 0 & $1(3 \%)$ \\
\hline $20-59$ & 4 & 0 & 0 & $4(13 \%)$ \\
\hline $60-79$ & 8 & 0 & 0 & $8(27 \%)$ \\
\hline$\geqslant 80$ & 12 & 2 & 2 & $16(53 \%)$ \\
\hline Unknown & 1 & 0 & 0 & $1(3 \%)$ \\
\hline \multicolumn{5}{|l|}{ Gender } \\
\hline Female & 18 & 0 & 0 & $18(60 \%)$ \\
\hline Male & 8 & 2 & 2 & $12(40 \%)$ \\
\hline
\end{tabular}

${ }^{*}$ One E. coli strain also harboured a non-ESBL $b l a_{\mathrm{TEM}}$ gene $\left(b l a_{\mathrm{TEM}-1}\right)$.

$\dagger$ One E. coli strain also harboured a non-ESBL $b l a_{\mathrm{TEM}}$ gene $\left(b l a_{\mathrm{TEM}-1}\right)$; the other E. coli strain also harboured an ampC gene (bla $a_{\mathrm{CMY}-2}$-like).

$\ddagger$ The two P. mirabilis strains also harboured a non-ESBL $b l a_{\mathrm{TEM}}$ gene $\left(b l a_{\mathrm{TEM}-1}\right)$. 
the Enterobacteriaceae in Emilia-Romagna. This finding confirms that CTX-Mproducing strains (mainly E. coli) are widespread throughout Italy [i.e. Varese, Bergamo, Novara, Verona, Florence, Ancona, Naples, Sassari, Palermo, Catania (Mugnaioli et al., 2006) and Pavia (Pagani et al., 2003)]. CTX-M-positive isolates have also been reported previously in Italy at the community level (Brigante et al., 2005) and in pets (Carattoli et al., 2005). The $b l a_{\mathrm{CTX}-\mathrm{M}-32}$ gene, retrieved in one strain cultured from the urine of an outpatient, is a novel variant derived from the $b l a_{\mathrm{CTX}-\mathrm{M}-1}$ gene through a single Asp240 $\rightarrow$ Gly substitution. Currently, isolates harbouring the $b l a_{\text {CTX-M-32 }}$ gene variant have emerged in some European countries such as Spain, Portugal and Italy, being associated with community-acquired infections (Cantón et al., 2008; LópezCerero et al., 2008).

Another finding deserving attention was the absence of $b l a_{\mathrm{TEM}}, b l a_{\mathrm{SHV}}$ and $b l a_{\mathrm{CTX}-\mathrm{M}}$ ESBL genes in the two ESBL-producing $P$. mirabilis isolates, suggesting the presence of unknown ESBL genes. In fact, PCR amplification of $b l a_{\mathrm{VEB}}, b l a_{\mathrm{PER}}$ and $b l a_{\mathrm{GES}}$ was also negative. Moreover, the identification of an E. coli strain carrying a $b l a_{\mathrm{CMY}}$-like gene highlights the presence of acquired AmpC-type $\beta$-lactamases in members of the Enterobacteriaceae, which represents another emerging problem in Italy (Migliavacca et al., 2007). Multifocal detection of $P$. mirabilis isolates carrying the $b l a_{\mathrm{CMY}-16}$ AmpC-encoding gene has been reported in different cities in northern Italy (i.e. Bergamo, Milan, Novara and Varese) (D'Andrea et al., 2006; Luzzaro et al., 2009).

Finally, the majority of ESBL-producing strains included in this study were also resistant to fluoroquinolones and/or aminoglycosides, with a consequent limited number of effective options for antibiotic treatment.

\section{Acknowledgements}

We gratefully thank Professor Gian Maria Rossolini for providing us with technical support for gene sequencing.

\section{Carlo Gagliotti, ${ }^{1}$ Mario Sarti, ${ }^{2}$ Franca Benini, ${ }^{3}$}

\section{Antonio Paolo Cipolloni, ${ }^{4}$ Claudia Venturelli, ${ }^{5}$ Carla Sabia, Raffaele Gargiulo ${ }^{2}$ and Maria Luisa Moro ${ }^{1}$}

${ }^{1}$ Agenzia Sanitaria e Sociale Regionale Emilia-Romagna, Viale Aldo Moro 21, 40127 Bologna, Italy

${ }^{2}$ AUSL Modena, Nuovo Ospedale di Modena S. Agostino-Estense, Via Giardini 1355, 41100 Baggiovara (MO), Italy

${ }^{3}$ AUSL Ravenna, Ospedale S. Maria Delle Croci, Viale Randi 5, 48100 Ravenna, Italy

${ }^{4}$ AUSL Cesena, Ospedale Bufalini, Viale Ghiotti 286, 47023 Cesena, Italy

${ }^{5}$ Azienda Ospedaliero-Universitaria di Modena, Via del Pozzo 71, 41100 Modena, Italy

${ }^{6}$ Università degli Studi di Modena e Reggio-Emilia, Dipartimento di Scienze Biomediche, Via Campi 287, 41100 Modena, Italy

Correspondence: Carlo Gagliotti (cgagliotti@regione.emilia-romagna.it)

Al Naiemi, N., Schipper, K., Duim, B. \& Bart, A. (2006). Application of minimal sequence values prevents misidentification of bla $a_{\mathrm{SHV}}$ type in single bacterial isolates carrying different SHV extended-spectrum $\beta$-lactamase genes. J Clin Microbiol 44, 1896-1898.

Brigante, G., Luzzaro, F., Perilli, M., Lombardi, G., Coli, A., Rossolini, G. M., Amicosante, G. \& Toniolo, A. (2005). Evolution of CTX-M-type beta-lactamases in isolates of Escherichia coli infecting hospital and community patients. Int $\mathrm{J}$ Antimicrob Agents 25, 157-162.

Cantón, R., Novais, A., Valverde, A., Machado, E., Peixe, L., Baquero, F. \& Coque, T. M. (2008).

Prevalence and spread of extended-spectrum beta-lactamase-producing Enterobacteriaceae in Europe. Clin Microbiol Infect 14 (Suppl. 1), 144153.

Carattoli, A., Lovari, S., Franco, A., Cordaro, G., Di Matteo, P. \& Battisti, A. (2005). Extendedspectrum beta-lactamases in Escherichia coli isolated from dogs and cats in Rome, Italy, from 2001 to 2003. Antimicrob Agents Chemother 49, 833-835.

CLSI (2007). Performance Standards for Antimicrobial Susceptibility Testing, 17th Informational Supplement. M100-S17. Wayne, PA: Clinical and Laboratory Standards Institute.

D'Andrea, M. M., Nucleo, E., Luzzaro, F., Giani, T., Migliavacca, R., Vailati, F., Kroumova, V.,
Pagani, L. \& Rossolini, G. M. (2006). CMY-16, a novel acquired AmpC-type $\beta$-lactamase of the CMY/LAT lineage in multifocal monophyletic isolates of Proteus mirabilis from Northern Italy. Antimicrob Agents Chemother 50, 618-624.

Gagliotti, C., Buttazzi, R., Capatti, C., Cassani, C., Lanzoni, M., Resi, D., Sarti, M., Venturelli, C. \& Moro, M. L. (2008a). Sorveglianza dell'Antibioticoresistenza e Uso di Antibiotici Sistemici in Emilia-Romagna. Rapporto 2006. Collana Dossier, no. 161. Bologna, Italy: Agenzia Sanitaria e Sociale Regionale EmiliaRomagna.

Gagliotti, C., Sarti, M., Benini, F., Cipolloni, A. P., Testa, G., Venturelli, C. \& Moro, M. L. (2008b). Laboratory detection of extended-spectrum beta-lactamase by an automated system. New Microbiol 31, 561-564.

López-Cerero, L., De Cueto, M., Saenz, C., Navarro, D., Velasco, C., Rodríguez-Baño, J. \& Pascual, A. (2008). Neonatal sepsis caused by a CTX-M-32-producing Escherichia coli isolate. J Med Microbiol 57, 1303-1305.

Luzzaro, F., Brigante, G., D'Andrea, M. M., Pini, B., Giani, T., Mantengoli, E., Rossolini, G. M. \& Toniolo, A. (2009). Spread of multidrugresistant Proteus mirabilis isolates producing an AmpC-type beta-lactamase: epidemiology and clinical management. Int J Antimicrob Agents 33, 328-333.

Migliavacca, R., Nucleo, E., D’Andrea, M. M., Spalla, M., Giani, T. \& Pagani, L. (2007). Acquired AmpC type beta-lactamases: an emerging problem in Italian long-term care and rehabilitation facilities. New Microbiol 30, 295-298.

Mugnaioli, C., Luzzaro, F., De Luca, F., Brigante, G., Perilli, M., Amicosante, G., Stefani, S., Toniolo, A. \& Rossolini, G. M. (2006). CTX-M-type extended-spectrum $\beta$-lactamases in Italy: molecular epidemiology of an emerging countrywide problem. Antimicrob Agents Chemother 50, 2700-2706.

Pagani, L., Dell'Amico, E., Migliavacca, R., D'Andrea, M. M., Giacobone, E., Amicosante, G., Romero, E. \& Rossolini, G. M. (2003). Multiple CTX-M-type extended-spectrum $\beta$-lactamases in nosocomial isolates of Enterobacteriaceae from a hospital in Northern Italy. J Clin Microbiol 41, 4264-4269.

Perilli, M., Dell'Amico, E., Segatore, B., De Massis, M. R., Bianchi, C., Luzzaro, F., Rossolini, G. M., Toniolo, A., Nicoletti, G. \& Amicosante, G. (2002). Molecular characterization of extended-spectrum $\beta$ lactamases produced by nosocomial isolates of Enterobacteriaceae from an Italian nationwide survey. J Clin Microbiol 40, 611-614.

Pitout, J. D. \& Laupland, K. B. (2008).

Extended-spectrum beta-lactamase-producing Enterobacteriaceae: an emerging public-health concern. Lancet Infect Dis 8, 159-166. 